\title{
An Adaptive Pulse Compression Filter for Ultrasound Contrast Harmonic Imaging
}

\author{
Jenho Tsao, Ming-Huang Chen \\ Graduate Institute of Biomedical Electronics and Bioinformatics, National Taiwan University \\ Email: tsaor215@cc.ee.ntu.edu.tw
}

Received December 2012

\begin{abstract}
Coded excitation is useful for ultrasound contrast imaging to increase penetration and SNR, and improve the contrast to tissue ratio (CTR). The waveform of bubble response depends greatly on bubble size, the frequency and bandwidth of the excitation chirp signal. This makes the pulse compression filter based on square-law be wrong for bubbles with changing sizes. In this paper, an adaptive pulse compression (APC) filter for the second harmonic of microbubble with varying size distribution is proposed. The APC filter is designed based on the estimated power spectrum of the received bubble harmonic echoes. Theoretical analysis and simulation studies are presented for evaluating performance of the APC filter. For monodisperse bubble, the power improvement factor of the APC filter can be more than $20 \mathrm{~dB}$.
\end{abstract}

Keywords: Ultrasound Contrast Imaging; Square Law Pulse Compression; Adaptive Pulse Compression

\section{Introduction}

Coded excitation is useful for ultrasound imaging to increase penetration and SNR. For improving the CTR (contrast to tissue ratio) in ultrasound contrast imaging, coded excitation is preferred also. In a previous research, Borsboom et al. suggest a nonlinear compression filter to do pulse compression for bubble second harmonic imaging [1]. They made use of a square law (SQL) model to design the pulse compression filter, which had the twice instantaneous frequency of the transmitted chirp.

The performance of microbubble agents depends on their resonant character to differ from tissue; the resonant character of bubble depends greatly on the size of bubble, and the frequency, bandwidth and waveform of the excitation signal. Novell et al. indicated that for up-sweep chirp, bubbles below resonance size provided a longer response; whereas bubbles above resonance size produced much more damped responses with large amplitude excursions [2]. Macdonald et al. showed that larger bubbles had more shift in resonance frequency than smaller bubbles. Also, the resonance frequency shifted to lower frequency when driving pressure was increased [3].

In this study, it is shown by simulation that chirp response of bubble depends greatly on the size of bubble and the frequency, bandwidth the excitation chirp signal. The pulse compression filter designed based on the SQL model (SPC) is a fixed pulse compression filter; equivalently it assumes that the chirp response of bubble is in-

*This work is supported by the National Science Council, Taiwan, ROC (NSC101-2221-E-002-081). variant. In reality, microbubbles have a time-varying size distribution in the perfusion region of interest, bubbles of different sizes respond to chirp excitation differently. This will lead to restrictions on the performance of the SPC filter. Consequently adaptive pulse compression (APC) is required to maximize the backscatter power of microbubbles for contrasting imaging to increase SNR.

In this paper, we propose an adaptive pulse compression filter to compress the second harmonic of bubble while microbubbles have a time varying size distribution. It is found that bandwidth of the second harmonic will be reduced while bubbles are excite to resonate. The APC filter is designed based on the estimated power spectrum of the received bubble harmonic echoes. Thus, it changes the pulse compression filter for bubble of different sizes adaptively to optimize the output power.

In the follows, theoretical analysis of the pulse compression problem is given in Section 2. The proposed adaptive pulse compression filter is given in Section 3. The performance of the APC filter is studied using simulation signals generated by BubbleSim [4]

\section{Theories}

Since the bubble response to chirp excitation is complicated, the theoretical foundations for the pulse compression problems are given below, step by step.

\subsection{Bubble Response}

The ultrasound contrast agents are encapsulated micro- 
bubbles, which provide strong scattering echo through the resonance behavior of bubbles. In general, bubble resonance is a complicated process, especially to drive the bubbles to generate high harmonics [4-6]. For a given driving pressure $x(t)$, the scattered pressure $y_{S}(t)$ can be found as

$$
y_{S}(t)=\rho_{l} \frac{R(t)}{r}\left(2 \dot{R}^{2}(t)+R(t) \ddot{R}(t)\right)
$$

where $R$ is the bubble radius, dynamics radius $R(t), r$ is the distance from bubble and $\rho_{l}$ is the liquid density [7]. The dynamics radius $R(t)$ can be found by solving the nonlinear Rayleigh-Plesset like differential equation:

$$
\begin{aligned}
& \rho\left(R \ddot{R}+\frac{3}{2} \dot{R}^{2}\right) \\
& =\left(p_{0}+\frac{2 \sigma}{R_{0}}\right)\left(\frac{R_{0}}{R}\right)^{3 \gamma}-\left(p_{0}+\frac{2 \sigma}{R_{0}}\right)-4 \mu \frac{\dot{R}}{R}+x(t)
\end{aligned}
$$

where $R_{0}$ and $R$ are the initial and instantaneous radius of the bubble, $\rho$ and $\mu$ are the density and viscosity of the liquid, $\sigma$ is the surface tension, $\gamma$ is the polytropic exponent, $p_{0}$ is the ambient pressure, and $x(t)$ is the incident pressure.

When driving pressure is a simple sinusoidal, the scattering cross section (SCS) can be found analytically as a function of driving frequency and bubble radius to be $\sigma_{s}^{(n)}(f, a)$ [4-7], where $n$ represents $n$-th harmonic. For complicated driving waveform, such as chirp, the only way to find the scattered pressure is by solving the Rayleigh-Plesset like equation. In this study, the chirp responses of bubbles are found by using BubbleSim [4] to solve the Rayleigh-Plesset like equation.

\subsection{The SCS of Microbubbles}

The scattering cross section of microbubbles provides an easy way to investigate the scattering properties of microbubbles. Based on Church's formulation, Equation (26b) in [6], for a microbubble coated with an elastic solid, its second-harmonic SCS can found to be a function of driving frequency and bubble radius. The bubble parameters used are: shell thickness $=4 \mathrm{~nm}$, shell shear modulus $=50 \mathrm{MPa}$, shell viscosity $=0.8$ Pas, ambient pressure $=100 \mathrm{KPa}$, polytropic exponent $=1$, viscosity of the liquid $=0.001$ Pas, density of the surrounding liquid $=1000 \mathrm{~kg} / \mathrm{m}^{3}$ and density of the shell material $=1100$ $\mathrm{kg} / \mathrm{m}^{3}$ [4].

The SCS's of the second harmonic of three bubbles with radius 1.5, 2.5 and $3.0 \mu \mathrm{m}$ are shown in Figure 1. Since bubbles are resonant scatters, and resonance is in general a narrow-band phenomenon, the SCS's have different resonant bandwidths. It can be found that smaller bubble has larger resonant bandwidth than that of larger bubble. The resonant bandwidth of the $3.0 \mu \mathrm{m}$ is quite

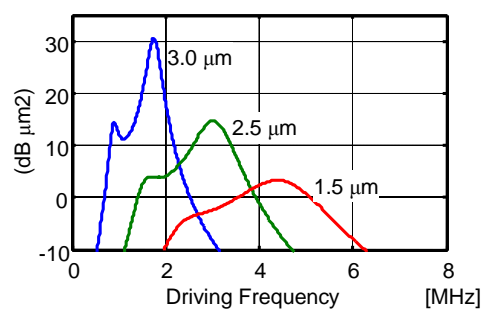

Figure 1. The scattering cross sections of three microbubbles.

small, which may not be good for imaging system to have high range resolution. Although SCS can not show the effect of resonant bandwidth on the scattered waveform, it does show the problem of insufficient bandwidth for large microbubbles. The apparent differences among the bubbles with different sizes also predict that their chirp responses will be very different. More detail about the chirp responses of microbubbles are given next.

\subsection{The Chirp Response}

The transmitted chirp signal is defined as:

$$
x(t)=\pi w(t) \cdot \cos \left(2\left(\frac{B}{2 T} t+f_{c}\right) t\right), \quad-\frac{T}{2} \leq t \leq \frac{T}{2}
$$

where $w(t)$ is a window function, $B$ is excitation bandwidth, $T$ is the signal duration defined by $w(t)$ and $f_{c}$ is center (driving) frequency [8]. In latter uses, the window function is a Hanning window discretized at 100 $\mathrm{MHz}$ and signal duration is $10 \mu \mathrm{s}$. The center frequency and bandwidth are set when they are used in different cases.

Based on square law, the second harmonic of microbubble is modeled to have a waveform as $x^{2}(t)[1,9$, 10]. Thus the pulse compression correlator has an impulse response as

$$
h_{S Q}(t)=w^{2}(t) \cdot \cos \left(4 \pi\left(\frac{B}{2 T} t+f_{c}\right) t\right)
$$

To exploit the properties of the scattered signal of bubbles excited by the chirp signal, BubbleSim is employed to solve a modified Raleigh-Plesset equation in [4] with parameters set same as in computing the SCS given in last section. The chirp signal is set to have bandwidth $=2 \mathrm{MHz}$ and transmission acoustic pressure $=50 \mathrm{KPa}$.

In Figure 2, the top panel shows the impulse response of the pulse compression correlator based on square-law, the others are the simulated waveforms of bubbles with radius $=1,2,3$ and $4 \mu \mathrm{m}$. Their waveforms are different significantly different from each other. Based on matched filter theory, the impulse response of a pulse compression filter should have same waveform as the scattered pressure to be compressed. However, the scattered pressures of bubbles with different radiuses are so different, it's 



Figure 2. The predicted and simulated waveforms. The SQL predicted waveform by square-law (Top panel) and the simulated waveforms of bubbles with radius $=1,2,3$ and 4 $\mu \mathrm{m}$.

almost impossible to have an ideal pulse compression filter. By comparing the waveforms in Figure 2, it can be found that the waveform predicted by the square-law (top panel) has high similarity to the scattered pressures of 1 and $2-\mu \mathrm{m}$ bubbles, but it is very bad for the 3 and $4-\mu \mathrm{m}$ bubbles. This shows that the pulse compression filter should be designed differently for bubbles with different sizes and predicts that there will be compression loss for the square-law pulse compression filter.

\section{The Adaptive Pulse Compression Filter}

The impulse response of the adaptive pulse compression filter (correlator) is defined as the chirp signal with parameters to be set adaptively:

$$
h_{A}(t)=w(t) \cdot \cos \left(2 \pi\left(\frac{B_{A}}{2 T_{A}} t+f_{A}\right) t\right), \quad-\frac{T_{A}}{2} \leq t \leq \frac{T_{A}}{2}
$$

The center frequency and bandwidth of APC, $f_{A}, B_{A}$, are the estimated center frequency and bandwidth of the received scattering signal of bubbles. The duration $T_{A}$ is set as $T_{A}=T B_{A} / B$, which affects the length of $w(t)$.

Performances of the proposed APC filter are studied using simulation signals of BubbleSim. Since the scat- tered pressure of bubble depend simultaneously on the driving frequency, excitation bandwidth and bubble size, the simulations are done for two driving frequencies, 2.45 and 3.5 MHz, and two excitation bandwidths, 2 and $4 \mathrm{MHz}$. The pulse compressions are done for bubbles with size ranged from 0.5 to $5 \mu \mathrm{m}$ by the square-law (SPC) and adaptive PC (APC) filters. Results are presented in Figures 3 to 5 . The top panels are the output powers of pulse compression done by SPC and by APC. The output powers of APC's are all larger than that of SPC's. The dB difference between APC and SPC are

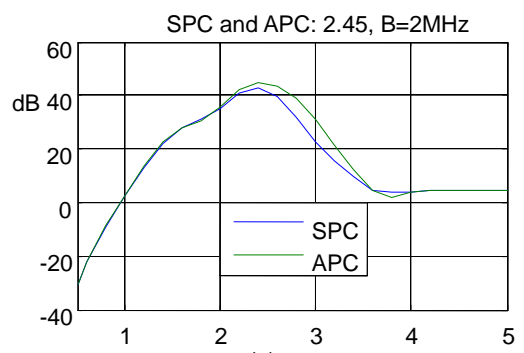

(a)

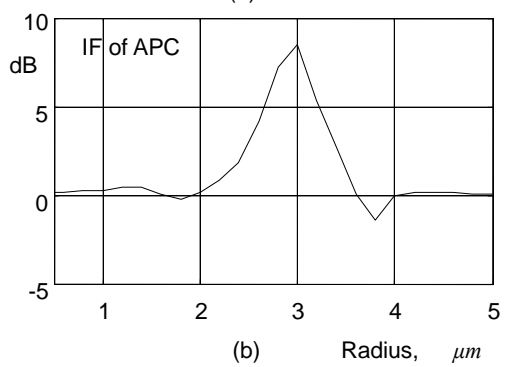

Figure 3. (a) Output power of pulse compression by squarelaw (SPC) and by adaptive PC (APC), for fc $=2.45 \mathrm{MHz}$ and B = 2 MHz; (b) The improvement factor of APC over SPC.

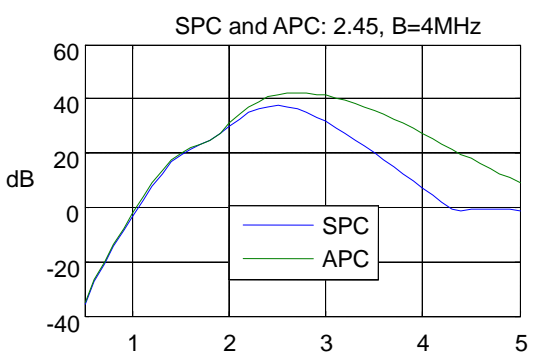

(a)

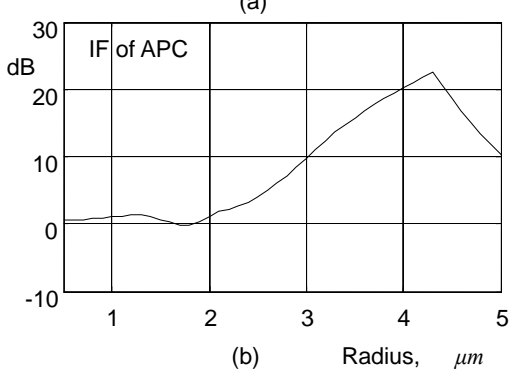

Figure 4. (a) Output power of pulse compression by squarelaw (SPC) and by adaptive PC (APC), for fc $=2.45 \mathrm{MHz}$ and $\mathrm{B}=4 \mathrm{MHz}$; (b) The improvement factor of APC over SPC. 

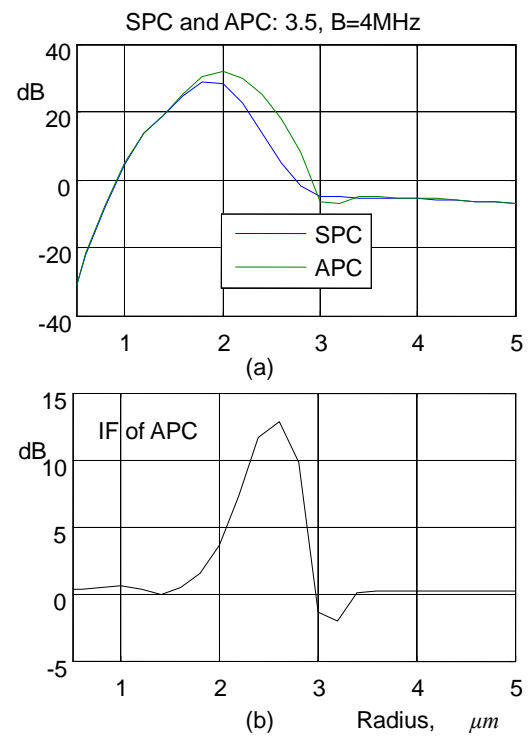

Figure 5. (a) Output power of pulse compression by squarelaw (SPC) and by adaptive PC (APC), for fc $=3.5 \mathrm{MHz}$ and $\mathrm{B}=4 \mathrm{MHz}$; (b) The improvement factor of APC over SPC.

defined as the improvement factor for the APC and shown in the lower panels. Two points found by comparing the three cases are given below.

1) All three cases show a same property that APC filter has power improvement over SPC only for bubbles within a range of sizes. That is the performance of APC is bubble-size dependent. This result has a close relation with the resonance property of bubble; for a given excitation signal, only bubbles with certain sizes can be excited to resonate. Too small or too large bubbles will not be excited to resonate, then there is no waveform distortion; in such case, SPC can predict the waveform of scattered pressure correctly and has a same result as APC.

2) The APC filter provides more improvement for larger excitation bandwidth. This property can be obtained by comparison that both Figures $\mathbf{4}$ and $\mathbf{5}$ has improvement factor (IF) larger than $20 \mathrm{~dB}$, however Figure 3 has IF around $8 \mathrm{~dB}$ only.

Based on the bubble-size dependent property of APC, it can be concluded that, for bubbles with a given size distribution, to have optimal power by PC, not only the PC filter must be designed adaptively, the driving frequency must be selected to match the size distribution also. In addition, the bubble-size dependent property of APC confirms that SPC is not proper for chirp harmonic imaging when bubbles are excited to resonate.

\section{Conclusions}

In this study, the square-law based pulse compression filter is shown to be improper for contrast harmonic imaging. Based on simulation studies, the performance of
APC is found to be bubble-size dependent due to bubble resonance. To have optimal power by APC, the driving frequency must be adapted to match the size distribution too. The APC filter is shown to be able to have a power improvement factor to be more than $20 \mathrm{~dB}$ over the SPC filter.

Since the resonant character of microbubble depends complicatedly on the size of bubble and the frequency, bandwidth and waveform of the excitation signal, more studies including experiments are necessary to prove the validity and performance of the APC filter.

\section{REFERENCES}

[1] J. M. G. Borsboom, C. T. Chin, A. Bouakaz, M. Versluis, and N. de Jong, "Harmonic Chirp Imaging Method for Ultrasound Contrast Agent," IEEE Transactions on Ultrasonics, Ferroelectrics and Frequency Control, Vol. 52, 2005, pp. 241-249.

http://dx.doi.org/10.1109/TUFFC.2005.1406550

[2] A. Novell, S. Van Der Meer, M. Versluis, N. De Jong, and A. Bouakaz, "Contrast Agent Response to Chirp Reversal: Simulations, Optical Observations, and Acoustical Verification," IEEE Transactions on Ultrasonics, Ferroelectrics and Frequency Control, Vol. 56, 2009, pp. 1199-1206. http://dx.doi.org/10.1109/TUFFC.2009.1161

[3] C. MacDonald, V. Sboros, et al., "A Numerical Investigation of the Resonance of Gas-Filled Microbubbles: Resonance Dependence on Acoustic Pressure Amplitude,” Ultrasonics, Vol. 43, 2004, pp. 113-122. http://dx.doi.org/10.1016/j.ultras.2004.04.001

[4] L. Hoff, "Acoustic Characterization of Contrast Agents for Medical Ultrasound Imaging," Kluwer Academic, Boston, 2001. http://dx.doi.org/10.1007/978-94-017-0613-1

[5] L. Hoff, P. C. Sontum and J. M. Hovem, "Oscillations of Polymeric Microbubbles: Effect of the Encapsulating Shell," Journal of the Acoustical Society of America, Vol. 107, 2000, pp. 2272-2280.

[6] C. C. Church, "The Effects of an Elastic Solid Surface Layer on the Radial Pulsations of Gas Bubbles,” Journal of the Acoustical Society of America, Vol. 97, 1995, pp. 1510-1421. http://dx.doi.org/10.1121/1.412091

[7] S. Hilgenfeldt, D. Lohse and M. Zomack, "Response of Bubbles to Diagnostic Ultrasound: A Unifying Theoretical Approach,” European Physical Journal B, Vol. 4, 1998, pp. 247-255. http://dx.doi.org/10.1007/s100510050375

[8] T. Misaridis and J. A. Jensen, "Use of Modulated Excitation Signals in Medical Ultrasound. Part II: Design and Performance for Medical Imaging Applications,” IEEE Transactions of UFFC, Vol. 52, 2005, pp. 192-207. http://dx.doi.org/10.1109/TUFFC.2005.1406546

[9] D. Y. Kim, J. C. Lee, S. J. Kwon and T.K. Song, "Ultrasound Second Harmonic Imaging with a Weighted Chirp Signal," Proceedings of IEEE Ultrasound Symposium, 2001, pp. 1477-1480.

[10] D. H. Simpson, C. T. Chin and P. N. Burns, "Pulse Inversion Doppler: A New Method for Detecting Nonlinear 
Echoes from Microbubble Contrast Agents,” IEEE Transactions on Ultrasonics, Fer- roelectrics and Frequency
Control, Vol. 46, 1999, pp. 372-382. http://dx.doi.org/10.1109/58.753026 